\title{
Criminologie
}

\section{Colonialisme et pollution environnementale} Prolongement et effets sur les droits des peuples autochtones

Colonialism and environmental pollution Prolongation and impacts on the human rights of indigenous peoples

\section{Colonialismo y contaminación medioambiental Prolongación y efectos solbre los derechos de los pueblos autóctonos}

\section{Doris Farget}

Volume 49, numéro 2, automne 2016

Criminalité environnementale

URI : https://id.erudit.org/iderudit/1038418ar

DOI : https://doi.org/10.7202/1038418ar

Aller au sommaire du numéro

Éditeur(s)

Les Presses de l’Université de Montréal

ISSN

0316-0041 (imprimé)

1492-1367 (numérique)

Découvrir la revue

Citer cet article

Farget, D. (2016). Colonialisme et pollution environnementale : prolongement et effets sur les droits des peuples autochtones. Criminologie, 49(2), 95-114. https://doi.org/10.7202/1038418ar
Résumé de l'article

Sachant que la contamination de l'environnement altère, limite ou élimine l'accès à certains domaines utilisés ou revendiqués par des peuples autochtones, notamment le territoire, la faune et la flore, quels sont les effets de la pollution de l'environnement sur les droits de ces peuples ? Dans le cadre de cet article, nous soutenons l'idée selon laquelle les effets de la pollution environnementale prolongent ou reproduisent ceux du colonialisme sur le plan des droits reconnus et de la normativité autochtone. C'est à travers cette lunette que nous analysons l'exercice ou l'accès à certains droits reconnus aux peuples autochtones dans un contexte de contamination environnementale, puis que nous analysons le type d'effet que celle-ci produit.
Tous droits réservés @ Les Presses de l’Université de Montréal, 2016
Ce document est protégé par la loi sur le droit d'auteur. L'utilisation des services d'Érudit (y compris la reproduction) est assujettie à sa politique d'utilisation que vous pouvez consulter en ligne.

https://apropos.erudit.org/fr/usagers/politique-dutilisation/ 


\title{
Colonialisme et pollution environnementale
}

\author{
Prolongement et effets sur les droits \\ des peuples autochtones
}

\author{
Doris Farget ${ }^{1}$ \\ Département d'anthropologie \\ Université McGill \\ dorisfarget@gmail.com
}

RÉSUMÉ - Sachant que la contamination de l'environnement altère, limite ou élimine l'accès à certains domaines utilisés ou revendiqués par des peuples autochtones, notamment le territoire, la faune et la flore, quels sont les effets de la pollution de l'environnement sur les droits de ces peuples? Dans le cadre de cet article, nous soutenons l'idée selon laquelle les effets de la pollution environnementale prolongent ou reproduisent ceux du colonialisme sur le plan des droits reconnus et de la normativité autochtone. C'est à travers cette lunette que nous analysons l'exercice ou l'accès à certains droits reconnus aux peuples autochtones dans un contexte de contamination environnementale, puis que nous analysons le type d'effet que celle-ci produit.

MOTS CLÉS - Autochtones, territoire, droits, environnement, pollution, colonialisme.

\section{Introduction}

Dans un contexte où la modernité prône la séparation de l'être humain, de la nature et de la culture (Descola, 2005; Latour, Schwartz et Charvolin, 1991), l'avènement de la révolution industrielle a densifié l'influence des êtres humains sur le système terrestre, la rendant prédominante (Latour, 2012). La pollution environnementale - c'est-à-dire les nuisances, la dégradation et la contamination par les déchets toxiques et les substances dangereuses de l'air, de l'eau, des sols et des êtres vivants - issue des activités humaines, notamment les activités

1. 2651, chemin Brunet, Sainte-Agathe-des-Monts (Québec), Canada, J8C $0 Z 7$.

Criminologie, vol. $49, \mathrm{n}^{\circ} 2$ (2016) 
industrielles, la construction de barrages hydroélectriques et l'extraction des ressources naturelles, a des répercussions sur les autres formes de vie et sur tous les êtres humains, peu importe leur origine et leur culture. Toutefois, les choix politiques, économiques, environnementaux et les activités industrielles ont des répercussions différentes en fonction des conditions sociales, culturelles et économiques des groupes sociaux. Associées à certains facteurs comme la pauvreté, la marginalisation sociale et politique ou l'origine ethnique, la pollution, les nuisances et la dégradation environnementale en touchent certains davantage (Agyeman, Cole et Haluza-Delay, 2009) 2.

Plusieurs raisons expliquent cette situation, notamment le fait que certains groupes sont plus exposés que d'autres à des activités polluantes, qu'ils sont établis à proximité de celles-ci ou qu'ils pratiquent des activités relatives à l'environnement (Lao Rhodes, 2003). Parmi ces groupes se trouvent les peuples autochtones ${ }^{3}$ (Johnson, 2009; Thériault, 2015). Bien que certains s'associent au développement industriel (Bousquet, à paraître), plusieurs d'entre eux en subissent aussi directement les conséquences néfastes (Working Group on Mining and Human Rights in Latin America, 2014) et font face à des rapports de pouvoir inégaux avec l'État et les compagnies (Papillon et Lord, 2013). La construction de barrages hydroélectriques, l'exploitation forestière et les déchets des usines de pâte à papier constituent, par exemple, des sources de pollution au mercure de l'eau potable, de la végétation ou de la faune (Ilyniak, 2014). L'usage du cyanure et d'autres produits toxiques par les compagnies minières contamine également les sols, les eaux et, de fait, la faune, la flore et l'être humain. La contamination du caribou par des déchets toxiques ou par les radiations nucléaires, l'altération de l'environnement par le stockage de déchets nucléaires, par les rayonnements ionisants et les radionucléides toxiques produits par l'exploitation de l'uranium, constituent d'autres exemples (AmabiliRivet, 2013). Malgré la variété des perspectives autochtones, l'hétérogénéité des situations, la diversité interne propre à chaque nation et la variété des conceptions de l'environnement, le lot de revendications

2. Sur l'histoire du mouvement pour la justice environnementale aux États-Unis, voir Johnson, 2009, p. 17-45.

3. Le concept de peuples autochtones n'a pas de définition officielle, ni en droit ni en sciences sociales. Des tentatives de définitions plus ou moins complexes existent au sein de la sphère juridique (notamment Cobo, 1986 et Kingsbury, 1998). Plusieurs auteurs en sciences sociales ont aussi développé des pistes de réflexion critique autour de ce concept et de sa définition (Barsh, 1994; Kenrick et Lewis, 2004; Tanner, 1992). 
partagées par ces nations à travers le monde et la mobilisation internationale de celles-ci autour du contrôle du territoire et de l'opposition à sa dégradation nous conduisent à penser que ces contaminations limitent ou altèrent, dans plusieurs cas, les pratiques d'utilisation du territoire, son occupation, la transmission des langues, de certains savoirs et de certaines conceptions, et ont donc des répercussions sur les droits, qu'ils soient d'applicabilité directe ou progressive. Partant de ce constat, quels sont les effets de la pollution de l'environnement sur les droits consacrés par les États ou sur les normes produites par les peuples autochtones ${ }^{4}$ ?

Certains ont déjà défendu la thèse selon laquelle les effets de la pollution environnementale prolongent ceux d'un colonialisme qualifié de «toxique» ou de "radioactif» (Churchill et LaDuke, 1983; Reed, 2009) et reproduisent les rapports de pouvoir et les inégalités sociales (McGregor, 2009). Dans cet article, nous nous inspirons de ces positions pour mener une réflexion exploratoire sur les effets de la pollution sur l'accessibilité des droits reconnus aux peuples autochtones. Nous allons d'abord exposer la reproduction des effets du colonialisme, des rapports de pouvoir et des inégalités sociales par la pollution environnementale (I), pour ensuite explorer la manifestation de ce prolongement sur le plan, d'une part, de l'accessibilité à certains droits possédés par les peuples autochtones au point de vue interne ou international et, d'autre part, des normativités autochtones (II). Sans considérer ces droits, les pratiques ou les savoirs qu'ils protègent, les normativités et les sociétés autochtones comme étant des «objets» figés et non susceptibles de transformation, nous soutenons que cette transformation, lorsqu'elle n'est pas recherchée ou consentie par le groupe qui la rencontre, révèle l'existence de rapports de pouvoir inégaux.

\section{Reproduction des effets du colonialisme, des rapports de pouvoir et des inégalités sociales par la pollution environnementale}

Dans cette partie, nous allons commencer par exposer ce qu'est le colonialisme tel qu'il a été notamment perpétré par les groupes dominants occidentaux, pour montrer que la pollution environnementale, notamment celle qui est liée à l'exploitation industrielle du territoire,

4. Pour évoquer ces normes, nous emploierons le concept de «normativité autochtone». 
prolonge et reproduit les effets de cette politique. Selon Aimé Césaire, poète, homme politique et fondateur du mouvement de la négritude:

colonisation $=$ chosification. J'entends la tempête. On me parle de progrès, de «réalisations», de maladies guéries, de niveaux de vie élevés au-dessus d'eux-mêmes. Moi, je parle de sociétés vidées d'elles-mêmes, de cultures piétinées, d'institutions minées, de terres confisquées, de religions assassinées, de magnificences artistiques anéanties, d'extraordinaires possibilités supprimées. (Césaire, 1955)

Pour Robert Lovelace (2009), le colonialisme est «[...] the process by which a group having exhausted its sustainability options dispossesses another group of its. Using seduction or force, a dominant group undermines the power of multiple others» (p. xix).

En contexte canadien, ces définitions sont bien illustrées par Rémi Savard. Cet anthropologue a notamment mis en évidence le fait que la colonisation suppose l'appropriation et la dépossession des terres, le déplacement de populations en fonction des besoins du colonisateur, l'assimilation de peuples au mode de vie et à la langue du colonisateur, la mise sous tutelle politique et l'invalidation, la stigmatisation de cultures, de langues et de normes, par l'entremise de politiques et de lois (Savard, 2002). En contexte canadien, ces actions et ces processus ont notamment été organisés par l'application, par la Couronne britannique, puis canadienne, d'une politique d'achat de terres, par la signature de traités comportant des clauses d'extinction des droits sur les terres, par la politique des pensionnats et par la Loi sur les Indiens (Savard, 2002).

Il en résulte que le colonialisme peut être associé à plusieurs caractéristiques, d'abord la dépossession territoriale et la perte de contrôle sur l'utilisation ou l'accès aux ressources naturelles qui constituent ce territoire, ensuite le fait de discréditer des normes, des savoirs, des conceptions, des visions du monde, des formes de spiritualité, des modes de gouvernance et des langues, mais aussi la marginalisation dans le processus de prise de décision.

En plus de faire courir des risques ou d'affaiblir la santé humaine, la pollution environnementale liée à l'exploitation industrielle du territoire conduit directement à l'altération partielle ou totale de l'environnement - c'est-à-dire du territoire, des êtres vivants et des ressources naturelles qui le composent. Ainsi, elle peut notamment contribuer à limiter l'accès à certains espaces, à transformer ou à réduire des pratiques, l'usage d'une langue et d'une terminologie. Elle peut transformer la 
mémoire collective, les repères et modifier ou éliminer certains lieux symboliques appartenant à l'histoire collective d'un groupe autochtone. De plus, la place qu'occupent ces groupes dans le processus de prise de décision portant sur les projets à risque de contamination, souvent plus symbolique qu'effective, révèle l'inégalité des positions dans la négociation avec l'État et les tiers, notamment les compagnies extractives. La marginalisation politique qui résulte de ce processus révèle des normes internes, des savoirs, des conceptions de l'environnement et un mode de vie qu'elle contribue à discréditer, sans toutefois les détruire (voir McGregor, 2004, p. 397-398). Le colonialisme, la dégradation de l'environnement par les activités extractives et les injustices environnementales et sociales qui en résultent ont donc plusieurs points communs. Selon McGregor (2009), "From the perspective of the world view within which I am embedded, environmental justice is most certainly about power relationships among people and between people and various institutions of colonization» (p. 27). De plus, le colonialisme et la dégradation de l'environnement par les politiques extractives sont fondés sur un récit commun, celui du progrès, promu par les tenants du modernisme et conduisant à invalider d'autres récits et d'autres conceptions du monde. Dans ce contexte, colonialisme, injustices sociales et environnementales proviennent tous deux du discrédit historique de certaines visions du monde. Par conséquent, la pollution environnementale, notamment celle qui est liée à l'exploitation industrielle du territoire et aux politiques extractives, prolonge les effets du colonialisme et reproduit les inégalités sociales (Ilyniak, 2014). Comment se manifeste ce prolongement sur le plan de l'effectivité des droits garantis et de la normativité autochtone? Autrement dit, quels peuvent en être les effets sur le plan de l'exercice des droits consacrés?

\section{Le prolongement des effets du colonialisme par la pollution environnementale sur le plan de l'exercice de certains droits et des normativités autochtones}

Nous avons rappelé que la pollution environnementale prolonge les effets du colonialisme et des inégalités sociales sur le plan de l'accès et de l'occupation du territoire, de l'utilisation de la faune, de la flore, des savoirs et de certaines terminologies relatives à des pratiques et à un vécu en territoire. La pollution environnementale a aussi plusieurs 
répercussions sur le plan juridique. Premièrement, elle peut reproduire une limitation de l'accès, de l'exercice ou de l'efficacité de certains droits étatiques reconnus spécifiquement aux peuples autochtones, à l'échelle interne et internationale. Autrement dit, outre les effets de la pollution sur la vie quotidienne, sur les pratiques et sur l'occupation du territoire, qui eux-mêmes exercent un effet domino sur la transmission des savoirs et des langues autochtones, la pollution environnementale peut empêcher l'accès à certains droits et leur pleine application, les vidant ainsi de leur substance (1). Deuxièmement, la pollution peut avoir pour effet de porter atteinte à la mise en œuvre de certains droits économiques et sociaux à l'égard des peuples autochtones (2). Troisièmement, la pollution environnementale révèle l'état actuel de marginalisation de certaines normes et valeurs autochtones au sein de la société, des politiques et du droit de l'État, notamment en ce qui concerne l'égalité de respect due aux êtres humains, aux êtres vivants et à la terre (Vincent, 1989). Toutefois, bien que les politiques économiques et extractives promues

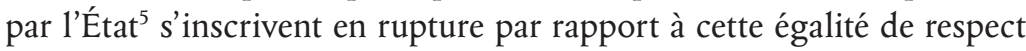
universelle, elles n'éteignent pas la validité de ces normes au sein des ordres juridiques autochtones (3).

\section{Les effets de la pollution environnementale sur l'accès et sur l'exercice de certains droits spécifiques}

La pollution environnementale a des effets sur l'accès et sur l'exercice de certains droits collectifs reconnus spécifiquement aux peuples autochtones par l'État canadien sur le plan interne ou sur le plan international. Sans éliminer la possibilité que la pollution environnementale affecte d'autres droits spécifiques, dans le cadre de cette réflexion exploratoire nous avons décidé de nous concentrer sur trois de ces droits: le droit au territoire, le droit des peuples autochtones à leur propre pharmacopée et le droit à leurs langues ${ }^{6}$.

5. Pour une définition de ces politiques, voir Svampa et Durand (2011).

6. Ces droits sont tous interdépendants et universellement consacrés aux peuples autochtones par la Déclaration des Nations Unies sur les droits des peuples autochtones. Certains, comme le droit au territoire, ont toutefois une applicabilité plus immédiate, parce que consacrés aussi par le droit interne. De plus, bien qu'ils soient tous enchâssés au sein d'un document international (la Déclaration des Nations Unies) qui a la nature d'une recommandation, non d'un traité international, une nature qui est plus politique que juridique, des auteurs considèrent que certaines dispositions de ce document tendent à obtenir une nature coutumière (Anaya, 2001). En outre, deux de ces droits, le droit au territoire et le droit à la langue sont aussi consacrés par les articles 14, 15 et 28 de la 
À l'échelle du droit international, plusieurs articles de la Déclaration des Nations Unies sur les droits des peuples autochtones [ci-après, la Déclaration des Nations Unies], signée en 2010 par le gouvernement canadien, protègent le droit de possession des peuples autochtones sur leurs terres, territoires traditionnels et ressources ainsi que le droit à la restitution des terres ${ }^{8}$. Certains tribunaux et quasi-tribunaux régionaux de protection des droits de la personne, comme la Cour interaméricaine des droits de l'homme et la Commission africaine des droits de l'homme et des peuples, se sont fondés sur ces dispositions pour reconnaître à des groupes autochtones un droit de propriété collective sur les terres et sur certaines ressources ou pour consacrer le droit à la restitution des terres $^{9}$. Sur le plan interne, l'article 35(1) de la Loi constitutionnelle de 1982 confirme les droits ancestraux des peuples autochtones ${ }^{10}$. Telle qu'elle est interprétée par la Cour suprême, cette disposition protège des pratiques et des traditions qui faisaient partie intégrante de la société du groupe requérant, avant le contact avec les Européens, comme le droit de pêcher à des fins alimentaires, sociales et rituelles, mais aussi des pratiques qui sont dans leur continuité, comme le commerce de la graisse d'eulakane ${ }^{11}$. Cette disposition protège aussi le titre aborigène, c'est-à-dire «le droit d'utiliser et d'occuper de façon exclusive les terres détenues en vertu de ce titre pour diverses fins», lesquelles ne se limitent pas aux utilisations traditionnelles ou «distinctives ${ }^{12} »$. En outre, en vertu de l'article 35 de la Loi constitutionnelle de 1982, le droit inhérent à l'autonomie gouvernementale pourrait permettre l'exercice d'un plus grand contrôle sur le territoire et ses ressources naturelles,

Convention 169 relative aux peuples indigènes et tribaux, dont la nature est celle d'un traité international. Celle-ci a d'ailleurs été ratifiée par plusieurs États d'Amérique du Sud, non par le Canada.

7. Énoncé du Canada appuyant la Déclaration des Nations Unies sur les droits des peuples autochtones, Affaires autochtones et Développement du Nord Canada, 12 novembre 2010, en ligne: https://www.aadnc-aandc.gc.ca/fra/1309374239861/1309374546142

8. Déclaration des Nations Unies sur les droits des peuples autochtones, Rés AG, Doc off AG NU, 61 ${ }^{e}$ sess, Doc NU A/RES/61/295 (2007), notamment les articles 26(1), 28 et 32 .

9. Notamment: Affaire Saramaka People (Suriname) (2007), Inter-Am Ct HR (Sér C) $\mathrm{n}^{\circ}$ 172, [2007] 2 Inter-American Yearbook on Human Rights 628, paragr. 131; Centre for Minority Rights Development (Kenya) and Minority Rights Group International on bebalf of Endorois Welfare Council v Kenya, 276/2003 (2010), Commission africaine des droits de l'homme et des peuples, paragr. 232.

10. Loi constitutionnelle de 1982, art 35(3), constituant l'annexe B de la Loi de 1982 sur le Canada (R-U), 1982, c 11.

11. Bande indienne des Lax Kw'alaams c. Canada (Procureur général), [2011] 3 RCS 535.

12. Nation Tsilhqot' in c. Colombie-Britannique, 2014 CSC 44, paragr. 70. 
lui-même fondé sur des savoirs et des normes propres (McGregor, 2004). Sur le plan strictement juridique, la Cour suprême du Canada n'a pas reconnu aux peuples autochtones l'existence d'un droit général à l'autonomie gouvernementale ${ }^{13}$, mais certains tribunaux de ColombieBritannique ont reconnu l'existence de ce droit dans une forme limitée (Leclair et Morin, 2011).

Ces dispositions internationales et internes garantissent des droits en matière d'accès, de contrôle, d'utilisation et d'occupation du territoire. Certaines reconnaissent aussi des droits de possession du territoire et d'autres, comme l'article 35(1) de la Loi constitutionnelle de 1982, protègent les Autochtones de certaines intrusions disproportionnées sur leurs territoires ancestraux de la part d'acteurs autorisés par l'État, qui exercent des activités comportant des risques de contamination. Toutefois, en vertu de cette disposition et de son interprétation générale donnée dans l'arrêt Nation Tsilhqot' in, «le gouvernement peut démontrer que l'atteinte est justifiée dans l'intérêt général du public ${ }^{14} »$. Les droits reconnus en vertu de cette disposition ne sont donc pas des droits absolus. Ils peuvent être limités si la Couronne poursuit un objectif impérieux et réel, si sa mesure se révèle être la moins attentatoire possible aux intérêts du groupe et si la Couronne a consulté au préalable le groupe autochtone affecté par sa mesure ${ }^{15}$. Rappelons aussi que les droits enchâssés au sein de la Déclaration des Nations Unies ont eux aussi une portée limitée, puisque cette résolution de l'Assemblée générale des Nations Unies a valeur de recommandation. En outre, sur le plan interne, les droits ancestraux et le titre aborigène protègent des pratiques et des traditions ancestrales et un droit d'accès au territoire, mais ne consacrent pas un droit général à la préservation des écosystèmes (Collins et Murtha, 2010). De plus, en pratique, les juges nationaux ont rejeté les requêtes de nombreux groupes autochtones qui recherchaient une garantie juridique de ces droits. D'autres groupes n'ont pas encore entamé les démarches devant les juges en raison du fait que la preuve de ces droits est longue et coûteuse à établir, en l'absence de documents écrits provenant des groupes autochtones et

13. R. c. Pamajewon, [1996] 2 R.C.S. 821.

14. Nation Tsilhqot' in c. Colombie-Britannique, 2014 CSC 44, paragr. 71.

15. R. c. Sparrow, [1990] 1 RCS 1075; Nation Haida c. Colombie-Britannique (Ministre des Forêts), [2004] 3 RCS 511 et Nation Tsilhqot' in c. Colombie-Britannique, 2014 CSC 44, paragr. 77. 
spécifiant les pratiques et la nature du titre avant ou au moment de la colonisation $^{16}$. Selon Leclair (2013):

[...] quand on sait que faire la démonstration d'un droit ancestral peut prendre des années, si raison était donnée aux provinces, il y aurait fort à parier que, le jour où un tribunal confirmerait l'existence du droit ancestral, le territoire serait déjà totalement déboisé ou le barrage hydroélectrique à moitié construit... Bref, si une communauté autochtone doit attendre de faire la démonstration d'un droit ancestral avant de pouvoir réclamer le droit d'être consulté, aussi bien dire que l'article 35 n'est, pour reprendre l'expression du baron de Lichtenberg, qu'un «couteau sans manche auquel manque une lame». (p. 312-313)

La protection juridique interne et internationale en matière d'occupation, d'utilisation, d'accès et de propriété sur le territoire a donc une portée tout à fait limitée et s'avère souvent inefficace pour protéger ces groupes contre les répercussions de la pollution.

Par conséquent, plusieurs groupes autochtones occupent leur territoire ancestral - des terres aujourd'hui qualifiées de domaniales, car appartenant à la Couronne - sans avoir de garanties juridiques ni de droits officiels d'occupation. La précarité juridique dans laquelle ces groupes se retrouvent peut avoir pour effet de limiter leur participation à la prise de décision concernant des projets comportant des risques de contamination des territoires ancestraux. Selon Agyeman et al. (2009), "Those with limited access to information, participatory opportunities, and/or the power to shape discourse(s) or decisions are less able to defend themselves and their communities from negative distributional environmental effects, and less prepared to advocate successfully for better environmental conditions» (p. 8). Autrement dit, l'absence de droits reconnus sur le territoire réduit d'autant plus la possibilité pour ces groupes d'encadrer et de «contenir» les activités à risque pouvant survenir sur leur territoire ancestral. Dans ce contexte, la pollution environnementale produite par ces activités peut non seulement révéler la situation de marginalisation politique de ces groupes, mais peut également avoir des effets limitatifs sur la revendication de droits ancestraux et sur les pratiques d'occupation et d'utilisation tolérées ou reconnues par l'État, par exemple, dans le cadre de

16. Jean Leclair qualifie le fardeau de preuve qu'ont les peuples autochtones du Canada sur le fondement de l'article 35(1) de la Loi constitutionnelle de 1982 "d'accablant» (Leclair, 2013, p. 308). 
droits exclusifs de trappe dans les réserves à castor ${ }^{17}$. Que des droits non absolus - sur le territoire et les ressources soient reconnus ou pas, la concrétisation d'un risque de contamination de l'environnement a pour effet, à court, moyen et long terme, de limiter l'occupation et les pratiques autochtones au sein des zones contaminées. Si elle se produit à long terme, cette limitation de l'occupation rend partiellement désuets la démarche d'obtention de droits ou l'exercice des droits reconnus. En la matière, plusieurs cas démonstratifs ont été documentés, dont celui de la communauté Anishinaabe de Grassy Narrows (Ilyniak, 2014) ou celui de la communauté innue de Sheshatshit au Labrador (Samson, 2003).

D'autres droits reconnus spécifiquement aux peuples autochtones subissent aussi ce type de limitations. Le droit à l'autodétermination interne des peuples autochtones, prévu à l'article 3 de la Déclaration des Nations Unies, en constitue un exemple. La pollution restreignant ou rendant infréquentable certains espaces sur lesquels ce droit pourrait s'appliquer, elle limite la mise en œuvre ou l'effectivité produite par ce droit.

Un autre exemple porte sur les droits des peuples autochtones à leur propre pharmacopée et à l'usage de leurs pratiques médicales protégés par l'article 24(1) de la Déclaration des Nations Unies. Bien qu'en vertu du second alinéa de cette disposition, ces droits semblent être d'applicabilité progressive et que les États soient responsables d'en assurer la réalisation graduellement, en fonction de leurs moyens, leur mise en œuvre, sans qu'elle puisse nuire aux droits et libertés fondamentales (article 46(2) et (3) de la Déclaration des Nations Unies), suppose notamment le respect de certains savoirs et la protection de la faune et de la flore. Comme le souligne un rapport de l'Assemblée des Premières Nations (2009):

Avant l'arrivée des Européens, les Autochtones pratiquaient une approche holistique de médecine et de guérison. Les femmes autochtones pouvaient devenir guérisseuses ou chamanes et jouer le rôle de sage-femme. Elles avaient des connaissances en nutrition, herboristerie, gynécologie, counseling et obstétrique. La colonisation a interdit les cérémonies, démonisé ces connaissances et détruit la manière holistique et traditionnelle de guérir. (p. 15)

17. Sur les droits exclusifs de trappe dans les réserves à castor, voir Morin, 2013, p. $60-64$. 
Sachant que la pollution environnementale peut nuire à l'objectif de «préservation des plantes médicinales, animaux et minéraux d'intérêt vital» enchâssé au sein de l'article 24(1) de la Déclaration, dans quelle mesure la contamination de certaines plantes et de certains médicaments ne vient-elle pas ajouter ou prolonger la «démonisation» de ces connaissances et, ce faisant, porter atteinte au plein exercice des droits à sa propre pharmacopée et à des pratiques médicales propres?

En dernier lieu, la question de l'anéantissement ou de la limitation de l'accès et de l'exercice de certains droits spécifiques se pose relativement au droit à la langue. Il semblerait que le droit des peuples autochtones à leur propre langue résulte de la protection établie par l'article 35(1) de la Loi constitutionnelle de 1982 (Poliquin, 2013; Richstone, 1989). De plus, bien que la mise en œuvre de ce droit et le caractère nécessaire de son application soient laissés à la discrétion des États en vertu de la Déclaration des Nations Unies (articles 13(2) et 14(3)), la juridicité de ce droit est en construction à l'échelle internationale. L'article 13(1) de la Déclaration des Nations Unies indique:

Les peuples autochtones ont le droit de revivifier, d'utiliser, de développer et de transmettre aux générations futures leur histoire, leur langue, leurs traditions orales, leur philosophie, leur système d'écriture et leur littérature, ainsi que de choisir et de conserver leurs propres noms pour les communautés, les lieux et les personnes.

Il n'est pas étonnant de constater que cette disposition ne protège pas seulement le droit des peuples autochtones à l'utilisation et à la transmission de leurs langues, mais aussi à leurs histoires, à leurs traditions, à leurs philosophies, à leurs littératures et à leurs propres toponymies, puisque tous ces éléments interagissent les uns avec les autres. Non seulement la langue en constitue le vecteur, mais elle contient une terminologie adaptée aux références et aux concepts centraux imbriqués dans la philosophie, dans les pratiques et les savoirs d'un groupe. Ce faisant, la langue construit et véhicule une conception du paysage local et du territoire. Selon Main Johnson (2011):

The characteristics of language, culture, local economy and the features of the biophysical world all contribute to local landscape terminology, toponyms and discourse about landscape, which in turn encodes knowledge about places and directs attention to significant features of the landscape. (p. 322)

En outre, l'article 14(1) de la Déclaration des Nations Unies protège le droit des peuples autochtones «d'établir et de contrôler leurs propres 
systèmes et établissements scolaires où l'enseignement est dispensé dans leur propre langue, d'une manière adaptée à leurs méthodes culturelles d'enseignement et d'apprentissage».

À la lecture des documents internationaux, l'accès à l'utilisation et à la transmission des langues autochtones semble donc être un objectif important, d'autant plus que la Convention 169 relative aux peuples indigènes et tribaux, adoptée en 1989 par l'Organisation internationale du travail, évoquait déjà cet enjeu à son article 28(1), sans toutefois impliquer directement les peuples autochtones dans sa réalisation. Or, la pratique d'une langue comporte aussi l'usage de toponymes associés au territoire - transmis de génération en génération ou effacés en raison de l'irruption de circonstances nouvelles ${ }^{18}$ - et de certains termes spécifiques désignant des phénomènes ou des manifestations propres à la forêt. Par conséquent, si contaminer un lieu a pour effet d'en limiter l'occupation et l'utilisation, cela a également pour effet de transformer, de limiter ou d'effacer l'usage de ces toponymes ou la transmission de termes spécifiquement liés à ce lieu et éventuellement de porter atteinte à la richesse des langues autochtones pratiquées dans cet espace, que ces langues et leur utilisation soient ou non promues par le droit. Le problème ne réside pas dans le fait que la langue se transforme, phénomène inévitable pour chacune d'entre elles. Il demeure plutôt dans le fait que bien des fois, cette transformation due aux effets des activités extractives n'a pas été choisie par les membres d'un groupe et qu'elle s'inscrit au sein de rapports inégaux, que ce soit avec l'État ou avec les compagnies.

\section{Effets sur certains droits économiques et sociaux}

Les droits économiques et sociaux examinés dans le cadre de ce texte sont le droit à la santé, le droit à un niveau de vie suffisant et à une alimentation saine, le droit à un environnement sain et le droit d'accès à une eau salubre non contaminée. Ces droits sont protégés à l'égard de tous les individus par les droits de la personne et certains s'appliquent spécifiquement aux peuples autochtones, lorsqu'ils sont protégés par la Déclaration des Nations Unies. Complémentaires aux droits civils et politiques, destinés à améliorer les conditions de vie des individus, en

18. À ce propos, le cas de la communauté Chippewas établie à côté de la ville de Sarnia est éloquent (voir Jackson, 2011, p. 610-611). 
particulier celles des plus marginalisés, ces droits sont toutefois d'applicabilité progressive en vertu du droit international.

Sur le plan interne, le droit à un environnement sain et le droit à un niveau de vie décent sont des droits justiciables au Québec en vertu de la Charte des droits et libertés de la personne ${ }^{19}$. De plus, en droit international, le droit à la santé, le droit à un niveau de vie suffisant et le droit à une alimentation saine sont protégés ${ }^{20}$. En contexte autochtone, l'article 29(1) de la Déclaration des Nations Unies protège le droit à un environnement sain. Le droit d'accès à une eau salubre non contaminée résulte quant à lui de l'interprétation faite par le Comité des droits économiques et sociaux des articles 11 et 12 du Pacte international relatif aux droits économiques et sociaux ${ }^{21}$.

Les effets de la pollution environnementale sur la santé des personnes sont reconnus par la communauté scientifique (notamment Wilson et Spengler, 1996). La violation du droit à la vie ou du droit à l'intégrité physique et morale par des activités polluantes et nuisibles est également reconnue par certains tribunaux, dont les cours européenne et interaméricaine des droits de l'homme (Duhaime, 2011). Ces violations sont aussi présentes, sinon plus, dans le cas des peuples autochtones, puisque plusieurs Autochtones pratiquent la chasse, la pêche, la trappe et la cueillette de plantes ou de petits fruits et consomment le produit de ces pratiques. Les effets spécifiques de la pollution sur la santé des personnes et l'environnement sont d'ailleurs dénoncés par plusieurs groupes autochtones ${ }^{22}$.

19. Charte des droits et libertés de la personne, L.R.Q., c. C-12. Sur la justiciabilité des droits économiques et sociaux en vertu de la Charte québécoise, voir Robitaille, 2011, p. $49-92$.

20. Pour le droit à la santé, voir le Pacte international relatif aux droits économiques et sociaux, article 12; Déclaration des Nations Unies sur les droits des peuples autochtones, article 24(2). Pour le droit à un niveau de vie suffisant et le droit à une alimentation saine, voir le Pacte international relatif aux droits économiques et sociaux, article 11.

21. Comité des droits économiques, sociaux et culturels, Observation générale ${ }^{\circ} 15$ : Le droit à l'eau (art. 11 et 12 du Pacte), Doc off NU, 29e sess, E/C.12/2002/11 (2002) aux paragr. $1,8,10,12$. Sur la justiciabilité des dispositions du Pacte international relatif aux droits économiques et sociaux, voir Texier, 2011, p. 481-487.

22. Par exemple, les effets nocifs de la construction de barrages hydroélectriques, notamment liés à la contamination des ressources halieutiques et du caribou forestier par le mercure, ont été dénoncés par la Corporation Nishipiminan (Ekuanitshit). Mémoire présenté au Bureau d'audiences publiques sur l'environnement dans le cadre du projet d'aménagement d'un complexe hydroélectrique sur la rivière Romaine par Hydro-Québec, 2008 , p. 2. 
La contamination d'une étendue d'eau ou d'une rivière à la suite de la construction d'un barrage hydroélectrique a des répercussions, notamment sur la faune et sur la flore environnantes. L'accumulation de mercure dans l'eau contamine la faune, comme le saumon ou le caribou forestier ${ }^{23}$, donc l'alimentation des personnes qui utilisent la rivière et le territoire à proximité. En cas de contamination de la rivière, de la faune et de la flore environnantes, la santé des personnes sera affectée et l'exercice ou l'efficacité du droit à une alimentation saine ainsi que du droit à la santé seront limités. De plus, cette contamination pourra avoir pour effet de transformer le régime alimentaire de ces personnes, modifiant par le fait même les pratiques d'utilisation du territoire.

On peut alors constater que la violation de certains droits économiques et sociaux a des répercussions sur l'exercice de certains droits spécifiquement reconnus aux peuples autochtones, notamment les droits d'utilisation et d'occupation du territoire consacrés dans le cadre des droits ancestraux. En outre, cela met en lumière, d'une part, les limites d'une pensée juridique sectorisée, qui vise à réglementer chaque domaine ou champ de la vie humaine en vase clos et, d'autre part, la légitimité de la pensée politique extractiviste au sein du droit. Actuellement, ces politiques et les activités susceptibles de nuire à l'environnement qui en résultent semblent davantage faire l'objet d'une articulation à d'autres secteurs du droit, notamment les droits de la personne, plutôt qu'être limitées ou interdites.

\section{Effets sur les ordres juridiques autochtones et sur les savoirs autochtones}

Précisons d'emblée que la pollution environnementale ne détruit pas les ordres juridiques autochtones, les savoirs, la philosophie et la conception du développement qu'ils sous-tendent. En effet, malgré le discrédit produit par les politiques extractives promues par les États à l'égard des normes et des savoirs autochtones, certains intellectuels autochtones montrent la conservation par ces peuples de leurs visions du développement et la perpétuation de la transmission des savoirs (McGregor, 2004), de leurs philosophies, de leurs conceptions et des normes propres, notamment à propos de la coexistence des êtres humains et des êtres non humains. Selon Blaser (2004): 
Indigenous communities do not just resist development, do not just react to state and market; they also sustain "life projects". Life projects are embedded in local histories; they encompass visions of the world and the future that are distinct from those embodied by projects promoted by state and markets. Life projects diverge from development in their attention to the uniqueness of people's experiences of place and self and their rejection of visions that claim to be universal. (p. 26)

Pour Lovelace (2009), membre de la Première Nation Ardoch et enseignant à 1'Université Queen's: «Our knowledge systems, although compromised, continue to form the structure and hold a vast content of information that supports sustainable living. Our epistemologies continue to bear witness to the goodness of humanity and the love of a Creator and beautiful Creation" (p. XIX). Bien qu'invalidés et affectés par la mise en œuvre des politiques extractives, plusieurs groupes autochtones continuent à revendiquer et à mobiliser leurs propres savoirs, normes et conceptions dans le cadre de stratégies d'affirmation.

Cependant, «In many Aboriginal cultures, rocks are persons, as are salmon and all of our relations» (Agyeman et al., 2009, p. 10). Outre le fait que la pollution environnementale peut nuire ou détruire des formes de vie qui, pour certains groupes autochtones, ont une personnalité juridique et qui représentent d'importants symboles dans les cosmologies et les normativités autochtones (Cruikshank, 2005; Kohn, 2013), dans certains cas, elle révèle surtout l'état de marginalisation de ces normes au sein de la société dominante - par exemple, l'égalité de respect dû aux êtres humains et aux êtres autres qu'humains -, de valeurs, de formes de spiritualité et de savoirs qui ont déjà été invalidés par le processus de colonisation (Borrows, 2002).

\section{Conclusion}

La pollution environnementale a pour effet de limiter, de nuire ou d'annihiler les droits d'accès et d'utilisation reconnus ou potentiels à un territoire et à ses ressources. Ce faisant, elle peut limiter l'usage d'une terminologie et la transmission de savoirs, notamment ceux qui sont liés aux pharmacopées autochtones, elles-mêmes fortement fondées sur l'environnement. De plus, la pollution environnementale révèle l'invalidation de certaines normes par la société dominante, notamment certaines normes issues des ordres juridiques autochtones, comme l'égalité de respect dû tant aux êtres humains qu'aux autres êtres vivants. Cette étude exploratoire des effets de la pollution sur les droits des 
peuples autochtones révèle enfin la priorité donnée par l'État et par son système juridique à un modèle de développement parmi d'autres. Ces enjeux mériteront d'être approfondis au sein d'analyses futures et plus systématiques.

En contribuant à la marginalisation politique et normative des peuples autochtones, en transformant les pratiques d'utilisation et d'occupation du territoire, en limitant l'usage d'une langue, en modifiant les repères territoriaux et en limitant la mémoire collective par la dégradation de certains lieux symboliques, la pollution environnementale, notamment celle qui provient des activités extractives ou industrielles sur le territoire, prolonge ou reproduit les effets du colonialisme, notamment à travers la perte d'accès ou les déplacements qu'elle peut occasionner. Elle révèle aussi l'existence de rapports de pouvoir souvent inégaux entre l'État, les compagnies extractives et les peuples autochtones.

\section{Références}

Agyeman, J., Cole, P. et Haluza-Delay, R. (2009). Speaking for Ourselves: Environmental Justice in Canada. Vancouver, Colombie-Britannique: UBC Press.

Amabili-Rivet, V. (2013). Impact de l'exploitation des mines d'uranium sur la santé humaine. (Mémoire de maîtrise, Université de Sherbrooke). Sherbrooke, Québec, Canada. Repéré à <https://www.usherbrooke.ca/environnement/ fileadmin/sites/environnement/documents/Essais_2013/AmabiliRivet_V_2013-09-12_.pdf>

Anaya, J. et Williams, R. A. (2001). The Protection of Indigenous Peoples' Rights over Lands and Natural Resources Under the Inter-American Human Rights System. Harvard Human Rights Journal, 14, 33-86.

Assemblée des Premières Nations. (2009). La santé environnementale et les femmes des premières nations. Rapport de recherche de l'Unité de la gestion de l'environnement. Repéré à < http://www.afn.ca/uploads/files/rp-enviro_health_ and_women-fr.pdf>

Barsh, R. L. (1994). Indigenous Peoples in the 1990s: From Object to Subject of International Law? Harvard Human Rights Journal, 7, 33-86.

Blaser, M. (2004). Life Projects: Indigenous Peoples' Agency and Development. Dans M. Blaser, H. Feit et G. McRea (dir.), In the Way of development. Indigenous Peoples, Life Projects and Globalization (p. 26-44). Londres, RoyaumeUni/ New York, NY: Zed Books.

Borrows, J. (2002). Recovering Canada: The Resurgence of Indigenous Law. Toronto, Ontario: University of Toronto Press.

Bousquet, M.-P. (à paraître). From passive consumers to entrepreneurs: Building a political context for economic development in an Anishinabe community, Qc. Dans K. Iankova (dir.), Indigenous People and Economic Development. Farnham, Québec: Gower Publishing Ltd. 
Césaire, A. (1955). Discours sur le colonialisme (1950, 2 édition). Paris, France: Présence africaine.

Churchill, W. et LaDuke, W. (1983). Native America: The Political Economy of Radioactive Colonialism. Dans W. Churchill (dir.), Critical issues in Native North America (vol. II) (p. 25-67). Copenhague, Danemark: IWGIA.

Cobo, J. R. M. (1986). Étude de la discrimination à l'encontre des populations autochtones. Repéré à http://undesadspd.org/IndigenousPeoples/Library Documents/Mart\% C3\% ADnezCoboStudy.aspx

Collins, L. M. et Murtha, M. (2010). Indigenous Environmental Rights in Canada. The Right to Conservation Implicit in Treaty and Aboriginal Rights to Hunt, Fish, and Trap. Alberta Law Review, 47(4), 959-991.

Cruikshank, J. (2005). Do Glaciers Listen? Vancouver, Colombie-Britannique: UBC Press.

Descola, P. (2005). Par-delà nature et culture. Paris, France: Éditions Gallimard.

Duhaime, B. (2011). L'OÉA et le Protocole de San Salvador: suivre les indications de la carte ou emprunter les chemins de traverse? Dans P. Bosset et L. Lamarche (dir.), Droit de cité pour les droits économiques, sociaux et culturels. La Charte québécoise en chantier (p. 363-404). Cowansville, Québec: Éditions Yvon Blais.

Ilyniak, N. (2014). Mercury Poisoning in Grassy Narrows: Environmental Injustice, Colonialism, and Capitalist Expansion in Canada. McGill Sociological Review, 4, 43-66.

Jackson, D. D. (2011). Scents of Place. The Dysplacement of a First Nations Community in Canada. American Anthropologist, 113(4), 608-618.

Johnson, G. S. (2009). Environmental Justice. A Brief History and Overview. Dans F. C. Steady (dir), Environmental Justice in the new Millennium: Global Perspective on Race, Ethnicity, and Human Rights (p. 17-45). New York, NY: Palgrave Macmillan.

Kenrick, J. et Lewis, J. (2004). Indigenous Peoples' Rights and the Politics of the Term 'Indigenous'. Anthropology Today, 20(2), 4-9.

Kingsbury, B. (1998). «Indigenous Peoples» in International Law : A Constructivist Approach to the Asian Controversy. The American Journal of International Law, 92(3), 414-457.

Kohn, E. (2013). How Forest Think. Toward an Antbropology beyond the Human. Berkeley/ Los Angeles, CA: University of California Press.

Lao Rhodes, E. (2003). Environmental Justice in America. Bloomington, IN University Press.

Latour, B. (2012). Enquête sur les modes d'existence. Une anthropologie des modernes. Paris, France: La Découverte.

Latour, B., Schwartz, C. et Charvolin, F. (1991). Crises des environnements: défis aux sciences humaines. Futur Antérieur, 6.

Leclair, J. (2013). Les droits ancestraux en droit constitutionnel canadien: quand l'identitaire chasse le politique. Dans A. Beaulieu, S. Gervais et M. Papillon (dir), Les Autochtones et le Québec: Des premiers contacts au Plan Nord (p. 299321). Montréal, Québec: Les Presses de l'Université de Montréal. 
Leclair, J. et Morin, M. (2011). Peuples autochtones et droit constitutionnel. Dans JurisClasseur Québec, collection "Droit public», Droit constitutionnel, fasc. 15, Montréal, Québec: LexisNexis.

Lovelace, R. (2009). Notes from Prison: Protecting Algonquin Lands from Uranium Mining. Dans J. Agyeman, P. Cole et R. Haluza-Delay (dir.), Speaking for Ourselves: Environmental Justice in Canada (p. ix-xix), Vancouver, Colombie-Britannique: UBC Press.

Main Johnson, L. (2011). Language, landscape and ethnoecology, reflection from northwestern Canada. Dans D. M. Mark (dir.), Landscape in Language Transdisciplinary Perspectives (p. 291-326). Amsterdam, Pays-Bas: John Benjamins Publishing Company.

McGregor, D. (2004). Coming Full Circle. Indigenous Knowledge, environment and our future. American Indian Quaterly, 28(3-4), 385-410.

McGregor, D. (2009). Honouring Our Relations: An Anishnaabe Perspective on Environmental Justice. Dans J. Agyeman, P. Cole et R. Haluza-Delay (dir.), Speaking for Ourselves: Environmental Justice in Canada (p. 27-41). Vancouver, Colombie-Britannique: UBC Press.

Morin, M. (2013). Propriétés et territoires autochtones en Nouvelle-France: i - Contrôle territorial et reconnaissance de territoires nationaux. Recherches amérindiennes au Québec, 43(2-3), 59-75.

Papillon, M. et Lord, A. (2013). Les traités modernes: vers une nouvelle relation? Dans A. Beaulieu, S. Gervais et M. Papillon (dir.), Les Autochtones et le Québec: Des premiers contacts au Plan Nord (p. 343-362). Montréal, Québec: Presses de l'Université de Montréal.

Poliquin, G. (2013). La protection d'une vitalité fragile: les droits linguistiques autochtones en vertu de l'article 35. Revue de droit de McGill, 58(3), 573-605.

Reed, T. V. (2009). Toxic Colonialism, Environmental Justice, and Native Resistance in Silko's Almanac of the Dead. MELUS, 34(2), 25-42.

Richstone, J. (1989). La protection juridique des langues autochtones au Canada. Dans P. Pupier et J. Woehrling (dir.), Language and the Law : Proceedings of the First Conference of the International Institute of Comparative Linguistic Law (p. 266-67). Montréal, Québec: Wilson \& Lafleur.

Robitaille, D. (2011). Pour une théorie de la justiciabilité substantielle et processuelle des droits économiques et sociaux. Dans P. Bosset et L. Lamarche (dir.), Droit de cité pour les droits économiques, sociaux et culturels. La Charte québécoise en chantier (p. 49-92). Cowansville, Québec: Éditions Yvon Blais.

Samson, C. (2003). A Way of Life that Does not Exist. Canada and the Extinguishment of the Innu. Londres, Royaume-Uni/New York, NY: Verso.

Savard, R. (2002). Les peuples américains et le système judiciaire canadien: Spéléologie d'un trou de mémoire. Revue canadienne droit et société, 17(2), 123-148.

Svampa M. et Durand, G. (2011). Néo-«développementisme» extractiviste, gouvernements et mouvements sociaux en Amérique latine. Problèmes d'Amérique latine, 81(3), 101-127.

Tanner, A. (1992). Le pouvoir et les peuples du quart monde. Antbropologies et sociétés, 16(3), 17-35. 
Texier, P. (2011). Le Pacte international relatif aux droits économiques, sociaux et culturels et son Protocole facultatif: vers une réelle indivisibilité des droits de l'homme? Dans P. Bosset et L. Lamarche (dir.), Droit de cité pour les droits économiques, sociaux et culturels. La Charte québécoise en chantier (p. 481-487). Cowansville, Québec: Éditions Yvon Blais.

Thériault, S. (2015). Justice environnementale et peuples autochtones: les possibilités et les limites du système interaméricain des droits de la personne. Revue québécoise de droit international, Hors-série, 129-148.

Vincent, S. (1989). Le patrimoine faunique et le territoire national. Dans A. Bissonnette et L. Sauvé (dir.), Peuples autocbtones de l'Amérique du Nord. De la réduction à la coexistence (p. 412-485). Québec: Télé-Université.

Van Woudenberg, G. (2004). Des femmes et de la territorialité». Début d'un dialogue sur la nature sexuée des droits des autochtones. Recherches amérindiennes au Québec, 34(3), 75-86.

Wilson R. et Spengler, J. (1996). Particles in Our Air: Concentrations and Health Effects. Cambridge, Royaume-Uni: Harvard University Press.

Working Group on Mining and Human Rights in Latin America. (2014). The impact of Canadian Mining in Latin America and Canada's Responsibility. Executive Summary of the Report submitted to the Inter-American Commission on Human Rights. Repéré à <http://dplf.org/sites/default/files/report_ canadian_mining_executive_summary.pdf $>$.

\section{Colonialism and environmental pollution: Prolongation and impacts on the human rights of indigenous peoples}

ABSTRACT - Environmental contamination alters, limits, or eliminates access to certain areas or items used or claimed by indigenous peoples, especially traditional lands, plants, and animals. In such circumstances, what are the effects of environmental degradation on indigenous rights? In this article, I provide support for the view that the effects of environmental contamination prolong or reproduce those of colonialism. Arguing from this perspective, I identify the rights of indigenous peoples that are affected by environmental contamination and analyze the effect such contamination has on these rights.

KEYWORDS - Aboriginal people, territory, rights, environment, pollution, colonialism.

\section{Colonialismo y contaminación medioambiental. Prolongación y efectos sobre los derechos de los pueblos autóctonos}

RESUMEN - Sabiendo que la contaminación del ambiente altera, limita o elimina el acceso a ciertos dominios utilizados o revindicados por los pueblos autóctonos, especialmente el territorio, la fauna y la flora, ¿cuáles son los efectos de la contaminación medioambiental sobre los derechos de dichos pueblos? En el marco del presente artículo, sostenemos la idea según la cual los efectos de la contaminación medioambiental 
prolongan o reproducen aquellos del colonialismo en lo que atañe a los derechos reconocidos y a la normatividad de los pueblos autóctonos. A través de esta perspectiva analizaremos el ejercicio o el acceso a ciertos derechos reconocidos a los aborígenes en un contexto de contaminación medioambiental, así como el tipo de efecto producido por ella.

PALABRAS CLAVE - Aborígenes, territorio, derechos, medioambiente, contaminación, colonialismo. 